\title{
On a smoother of discrete-time linear stochastic systems with unknown disturbances
}

\author{
Akio Tanikawa \\ Faculty of Information Science and Technology \\ Osaka Institute of Technology \\ Kitayama, Hirakata-shi, 573-0196 Japan \\ tanikawa@is.oit.ac.jp
}

\begin{abstract}
We consider discrete-time linear stochastic systems with unknown disturbances and study a smoothing problem for those systems. We derive a smoothing algorithm by applying the optimal filter obtained in our previous work to an augmented system.
\end{abstract}

Keywords: stochastic systems, smoother, optimal filter, unknown inputs.

\section{Introduction}

We consider optimal filtering and smoothing problems for discrete-time linear stochastic systems. It is well known that a standard Kalman filter is the optimal linear filter since it minimizes the mean-square errors in an appropriate class of linear filters (see e.g., [14], [16], [17], [18] and [19]). We need to recall that the Kalman filter can work well only when we have an accurate mathematical modelling of the monitored system. However, in practice, models derived by engineers often contain modelling errors which greatly increase the state estimation error as if the models have unknown disturbances.

In order to develop reliable filtering algorithm which are robust with respect to unknown disturbances and modelling errors, many research works based on the use of the disturbance decoupling principle have been published. Pioneering works were done by Darouach et al. ([8] and [9]), Chang and Hsu ([4]) and Hou and Müller ([11]). They utilized some transformations to make the original systems with unknown inputs into some singular systems without unknown inputs. The most important previous study related to this paper was done by Chen and Patton ([5]). They proposed a simple and useful optimal filtering algorithm, ODDO (Optimal Disturbance Decoupling Observer), with unknown input decoupling property and showed excellent simulation results. See also the papers such as [3], [12], [13] and [22]. Their algorithm recently has been modified by the authors in [23].

The optimal filtering problem is to investigate the optimal estimate of the state $x_{t}$ or $x_{t+1}$ with min- imum variance based on the observation $\mathcal{Y}_{t}$ of the states $\left\{y_{0}, y_{1}, \cdots, y_{t}\right\}$, i.e., $\mathcal{Y}_{t}=\sigma\left\{y_{s}, s=0,1, \cdots, t\right\}$ ( the smallest $\sigma$-field generated by $\left\{y_{0}, y_{1}, \cdots, y_{t}\right\}$ (see e.g., Katayama [19], Chapter 4)), but we here consider smoothing problems which allow us time-lags for computing state estimates. Namely, we study the optimal estimate $\hat{x}_{t-L / t}$ of the state $x_{t-L}$ based on the observation $\mathcal{Y}_{t}$ with $L>0$. The smoothing problems are usually classified into the following three types. The first problem is the fixed-point smoothing for which we investigate the optimal estimate of the state $x_{k}$ for a fixed $k$ based on the observations $\left\{\mathcal{Y}_{t}, t=k+1, k+2, \cdots\right\}$. Algorithms to compute $\hat{x}_{k / t}, t=k+1, k+2, \cdots$, recursively are called fixed-point smoothers. The second problem is to study the optimal state estimate of $x_{t-L}$ based on the observation $\mathcal{Y}_{t}$ for a given $L \geq 1$. Algorithms to compute $\hat{x}_{t-L / t}, t=L+1, L+2, \cdots$, recursively are called fixed-lag smoothers. The third problem is to study the optimal state estimate $\hat{x}_{t / N}$ of the states $x_{t}$ at all times $t=0,1, \cdots, N$ based on the observation $\mathcal{Y}_{N}$ of all the states $\left\{y_{0}, y_{1}, \cdots, y_{N}\right\}$. Algorithms to compute $\hat{x}_{t / N}, t=N, N-1, \cdots, 0$ recursively are called fixed-interval smoothers. See also [1], [2], [15], [19] and [20] for smoothers.

We state some preliminary results in Section 2, and propose a fixed-point smoother in Section 3. This smoothing algorithm is derived by applying the optimal filter (see Proposition 2.2) obtained in [23] to an augmented system. We note that the fixed-point smoother is rather important also due to the reason why the fixedinterval smoother can be readily derived from the fixedpoint smoother. It will be discussed in the subsequent paper.

\section{Preliminaries}

Consider the following discrete-time linear stochastic system for $t=0,1,2, \cdots$ :

$$
\begin{aligned}
x_{t+1} & =A_{t} x_{t}+B_{t} u_{t}+E_{t} d_{t}+\zeta_{t}, \\
y_{t} & =C_{t} x_{t}+\eta_{t},
\end{aligned}
$$

where

$x_{t} \in \mathbf{R}^{n}$ the state vector 


$$
\begin{aligned}
& y_{t} \in \mathrm{R}^{m} \text { the output vector } \\
& u_{t} \in \mathbf{R}^{r} \text { the known input vector } \\
& d_{t} \in \mathbf{R}^{q} \text { the unknown input vector }
\end{aligned}
$$

$\zeta_{t}$ and $\eta_{t}$ are independent zero mean white noise sequences with covariance matrices $Q_{t}$ and $R_{t} . A_{t}, B_{t}, C_{t}$ and $E_{t}$ are known matrices with appropriate dimensions.

In [23], we studied the optimal state estimate $\hat{x}_{t+1 / t+1}$ of the state $x_{t+1}$ proposed by Chen and Patton ([5]) with the following structure:

$$
\begin{aligned}
& z_{t+1}=F_{t+1} z_{t}+T_{t+1} B_{t} u_{t}+K_{t+1} y_{t}, \\
& \hat{x}_{t+1 / t+1}=z_{t+1}+H_{t+1} y_{t+1}
\end{aligned}
$$

for $t=0,1,2, \cdots$. Let $\hat{x}_{0 / 0}$ be chosen to be $z_{0}$ for a fixed $z_{0}$. We denote the state estimation error by $e_{t}$, i.e., $e_{t}=x_{t}-\hat{x}_{t / t}$ for $t=0,1,2, \cdots$. Its covariance matrix (i.e., the error covariance matrix of state estimate (3)-(4)) is denoted by $P_{t}$, i.e., $P_{t}=\mathbb{E}\left\{e_{t} e_{t}^{T}\right\}$, where $\mathrm{E}$ denotes expectation and $T$ denotes transposition of a matrix. In this paper, we assume that random variables $e_{0},\left\{\eta_{t}\right\},\left\{\zeta_{t}\right\}$ are independent. Following Chen and Patton ([5]), we considered state estimate (3)-(4) with the matrices $F_{t+1}, T_{t+1}, H_{t+1}$ and $K_{t+1}$ of the forms ([23]):

$$
\begin{aligned}
& K_{t+1}=K_{t+1}^{1}+K_{t+1}^{2}, \\
& E_{t}=H_{t+1} C_{t+1} E_{t}, \\
& T_{t+1}=I-H_{t+1} C_{t+1}, \\
& F_{t+1}=A_{t}-H_{t+1} C_{t+1} A_{t}-K_{t+1}^{1} C_{t}, \\
& K_{t+1}^{2}=F_{t+1} H_{t} .
\end{aligned}
$$

The next lemma on equality (6) was obtained and used by Chen and Patton [5]. Before stating it, we assume that $E_{k}$ is a full column rank matrix which is not an essential restriction.

\section{Lemma 2.1 Equality (6) holds if and only if}

$$
\operatorname{rank}\left(C_{t+1} E_{t}\right)=\operatorname{rank}\left(E_{t}\right) .
$$

When this condition holds true, matrix $H_{t+1}$ which satisfies (6) must have the form

$$
\begin{aligned}
H_{t+1}=E_{t}\left\{\left(C_{t+1} E_{t}\right)^{T}\left(C_{t+1} E_{t}\right)\right\}^{-1} \\
\times\left(C_{t+1} E_{t}\right)^{T} .
\end{aligned}
$$

Hence, we have

$$
\begin{aligned}
C_{t+1} H_{t+1} & =C_{t+1} E_{t}\left\{\left(C_{t+1} E_{t}\right)^{T}\right. \\
& \left.\times\left(C_{t+1} E_{t}\right)\right\}^{-1}\left(C_{t+1} E_{t}\right)^{T}
\end{aligned}
$$

which is a non-negative definite symmetric matrix.

We found out the explicit form of the matrix $K_{t+1}^{1}$ which makes the variance of the state estimation error $e_{t+1}$ minimum and its sufficient conditions in ([23]). Thus, we considered the matrix $K_{t+1}^{1}$ given by

$$
\begin{aligned}
K_{t+1}^{1}= & A_{t+1}^{1}\left(P_{t} C_{t}^{T}-H_{t} R_{t}\right) \\
& \times\left(C_{t} P_{t} C_{t}^{T}+R_{t}\right)^{-1},
\end{aligned}
$$

where

$$
A_{t+1}^{1}=A_{t}-H_{t+1} C_{t+1} A_{t} .
$$

We here state the main result (Theorem 2.7 in ([23]) on the optimal filtering algorithm as follows.

Proposition 2.2 If $C_{t} H_{t}$ and $R_{t}$ are commutative, i.e.,

$$
C_{t} H_{t} R_{t}=R_{t} C_{t} H_{t}
$$

then the optimal gain matrix $K_{t+1}^{1}$ which makes the variance of the state estimation error $e_{t+1}$ minimum is determined by (13). Hence, we obtain the optimal filtering algorithm:

$$
\begin{gathered}
\hat{x}_{t+1 / t+1}=A_{t+1}^{1}\left\{\hat{x}_{t / t}+G_{t}\left(y_{t}-C_{t} \hat{x}_{t / t}\right)\right\} \\
+H_{t+1} y_{t+1}+T_{t+1} B_{t} u_{t} \\
P_{t+1}=A_{t+1}^{1} M_{t} A_{t+1}^{1}+T_{t+1} Q_{t} T_{t+1}^{T} \\
+H_{t+1} R_{t+1} H_{t+1}^{T}
\end{gathered}
$$

where

$$
G_{t}=\left(P_{t} C_{t}^{T}-H_{t} R_{t}\right)\left(C_{t} P_{t} C_{t}^{T}+R_{t}\right)^{-1},
$$

and

$$
M_{t}=P_{t}-G_{t}\left(C_{t} P_{t}-R_{t} H_{t}^{T}\right) .
$$

Remark 2.3 If the matrix $R_{t}$ has the form

$$
R_{t}=r_{t} I
$$

with some positive number $r_{t}$ for each $t=1,2, \cdots$, then condition (15) holds.

Finally, we remark that the standard Kalman filter is a special case of the optimal filter proposed in this section (see e.g., Theorem 5.2 (page 90) in Katayama [19]).

Proposition 2.4 Suppose that $E_{t} \equiv O$ holds for all $t$ (i.e., the unknown input term is zero). Then, Lemma 2.1 cannot be applied directly. But, we can choose $H_{t} \equiv O$ for all $t$ in this case, and the optimal filter given in Proposition 2.2 reduces to the standard Kalman filter.

\section{The fixed-point smoothing}

Let $k$ be a fixed time. We study an iterative algorithm to compute the optimal estimate $\hat{x}_{k / t}$ of the state $x_{k}$ based on the observation $\mathcal{Y}_{t}, t=k+1, k+2, \cdots$, where $\mathcal{Y}_{t}=\sigma\left\{y_{s}, s=0,1, \cdots, t\right\}$. We define state vectors $\theta_{t}, t=k, k+1, \cdots$, by

$$
\theta_{t+1}=\theta_{t}, t=k, k+1, \cdots ; \theta_{k}=x_{k} .
$$

It is easy to observe that the optimal estimate $\hat{\theta}_{t / t}$ of the state $\theta_{t}$ based on the observation $\mathcal{Y}_{t}$ is identical to the optimal smoother $\hat{x}_{k / t}$ in view of the equalities $\theta_{t}=x_{k}, t=k, k+1, \cdots$.

In order to derive the optimal fixed-point smoother, we consider the following augmented system for $t=$ 
$k, k+1, \cdots:$

$$
\begin{gathered}
{\left[\begin{array}{c}
x_{t+1} \\
\theta_{t+1}
\end{array}\right]=\left[\begin{array}{cc}
A_{t} & O \\
O & I
\end{array}\right]\left[\begin{array}{c}
x_{t} \\
\theta_{t}
\end{array}\right]+\left[\begin{array}{c}
B_{t} \\
O
\end{array}\right] u_{t}} \\
+\left[\begin{array}{c}
E_{t} \\
O
\end{array}\right] d_{t}+\left[\begin{array}{c}
I \\
O
\end{array}\right] \zeta_{t}, \\
y_{t+1}=\left[\begin{array}{ll}
C_{t+1} & O
\end{array}\right]\left[\begin{array}{c}
x_{t+1} \\
\theta_{t+1}
\end{array}\right]+\eta_{t+1} .
\end{gathered}
$$

Denote these equations respectively by

$$
\begin{aligned}
\widetilde{x_{t+1}} & =\widetilde{A_{t}} \widetilde{x_{t}}+\widetilde{B_{t}} u_{t}+\widetilde{E_{t}} d_{t}+\widetilde{J}_{t} \zeta_{t}, \\
y_{t+1} & =\widetilde{C_{t+1}} \widetilde{x_{t+1}}+\eta_{t+1},
\end{aligned}
$$

where

$$
\begin{aligned}
& \widetilde{x_{t}}=\left[\begin{array}{c}
x_{t} \\
\theta_{t}
\end{array}\right], \widetilde{A_{t}}=\left[\begin{array}{cc}
A_{t} & O \\
O & I
\end{array}\right], \widetilde{B_{t}}=\left[\begin{array}{c}
B_{t} \\
O
\end{array}\right], \\
& \widetilde{E_{t}}=\left[\begin{array}{c}
E_{t} \\
O
\end{array}\right], \widetilde{J_{t}}=\left[\begin{array}{l}
I \\
O
\end{array}\right] \text { and } \widetilde{C_{t+1}}=\left[\begin{array}{ll}
C_{t+1} & O
\end{array}\right] .
\end{aligned}
$$

Here, $I$ and $O$ are the identity matrix and the zero matrix respectively with appropriate dimensions. By making use of the notations

$$
\widetilde{H_{t+1}}=\left[\begin{array}{c}
H_{t+1} \\
O
\end{array}\right], \widetilde{T_{t+1}}=\left[\begin{array}{cc}
I & O \\
O & I
\end{array}\right]-\widetilde{H_{t+1}} \widetilde{C_{t+1}}
$$

we have the equalities:

$$
\begin{aligned}
& \widetilde{C_{t+1}} \widetilde{E_{t}}=C_{t+1} E_{t}, \\
& \widetilde{T_{t+1}}=\left[\begin{array}{cc}
T_{t+1} & O \\
O & I
\end{array}\right], \\
& \widetilde{A_{t+1}^{1}}=\widetilde{T_{t+1}} \widetilde{A_{t}}=\left[\begin{array}{cc}
A_{t+1}^{1} & O \\
O & I
\end{array}\right] .
\end{aligned}
$$

We introduce the covariance matrix $\widetilde{P}_{t}$ of the state estimation error of the augmented system (23)-(24):

$$
\begin{aligned}
\widetilde{P}_{t} & =\left[\begin{array}{ll}
P_{t}^{(1,1)} & P_{t}^{(1,2)} \\
P_{t}^{(2,1)} & P_{t}^{(2,2)}
\end{array}\right] \\
& =\mathbf{E}\left\{\left[\begin{array}{c}
x_{t}-\hat{x}_{t / t} \\
\theta_{t}-\hat{\theta}_{t / t}
\end{array}\right]\left[\begin{array}{c}
x_{t}-\hat{x}_{t / t} \\
\theta_{t}-\hat{\theta}_{t / t}
\end{array}\right]^{T}\right\} .
\end{aligned}
$$

Notice that $P_{t}^{(1,1)}$ is equal to $P_{t}$. Applying the optimal filter given in Proposition 2.2 to the augmented system (21)-(22), we obtain the following optimal fixed-point smoother.

Theorem 3.1 If $C_{t} H_{t}$ and $R_{t}$ are commutative, i.e.,

$$
C_{t} H_{t} R_{t}=R_{t} C_{t} H_{t},
$$

then we have the optimal fixed-point smoother for (21)(22) as follows:

(i) the fixed-point smoother

$$
\hat{x}_{k / t+1}=\hat{x}_{k / t}+D_{t}(k)\left[y_{t}-C_{t} \hat{x}_{t / t}\right],
$$

(ii) the gain matrix

$$
D_{t}(k)=P_{t}^{(2,1)} C_{t}^{T}\left(C_{t} P_{t} C_{t}^{T}+R_{t}\right)^{-1},
$$

(iii) the covariance matrix of the mean-square error

$$
\begin{aligned}
P_{t+1}^{(2,1)}= & \left\{P_{t}^{(2,1)}-P_{t}^{(2,1)} C_{t}^{T}\left(C_{t} P_{t} C_{t}^{T}+R_{t}\right)^{-1}\right. \\
& \left.\times\left(C_{t} P_{t}-R_{t} H_{t}^{T}\right)\right\} A_{t+1}^{1}{ }^{T} \\
P_{t+1}^{(2,2)}= & P_{t}^{(2,2)}-P_{t}^{(2,1)} C_{t}^{T}\left(C_{t} P_{t} C_{t}^{T}+R_{t}\right)^{-1} \\
& \times C_{t} P_{t}^{(2,1)^{T}} .
\end{aligned}
$$

Here, we note that $P_{k}^{(2,1)}=P_{k}^{(2,2)}=P_{k}$. We notice that $\hat{x}_{t / t}$ is the optimal filter of the original system (1)-(2) given in [23].

Proof Applying the optimal filter given by (16)-(17) in Proposition 2.2 to the augmented system (23)-(24), we have

$$
\begin{aligned}
x_{t+1 / t+1}=\widetilde{A_{t+1}} & \left\{\widehat{\widehat{x_{t / t}}}+\widetilde{G_{t}}\left(y_{t}-C_{t} \widehat{\widehat{x_{t / t}}}\right)\right\} \\
& +\widetilde{H_{t+1}} y_{t+1}+\widetilde{T_{t+1}} \widetilde{B_{t}} u_{t} .
\end{aligned}
$$

This can be rewritten as

$$
\begin{aligned}
& {\left[\begin{array}{c}
\hat{x}_{t+1 / t+1} \\
\hat{\theta}_{t+1 / t+1}
\end{array}\right]} \\
& =\left[\begin{array}{cc}
A_{t+1}^{1} & O \\
O & I
\end{array}\right]\left\{\left[\begin{array}{l}
\hat{x}_{t / t} \\
\hat{\theta}_{t / t}
\end{array}\right]+\left[\begin{array}{c}
P_{t}^{(1,1)} C_{t}^{T}-H_{t} R_{t} \\
P_{t}^{(2,1)} C_{t}^{T}
\end{array}\right]\right. \\
& \left.\quad \times\left(C_{t} P_{t} C_{t}^{T}+R_{t}\right)^{-1}\left(y_{t}-C_{t} \hat{x}_{t / t}\right)\right\} \\
& +\left[\begin{array}{c}
H_{t+1} y_{t+1} \\
O
\end{array}\right]+\left[\begin{array}{c}
T_{t+1} B_{t} u_{t} \\
O
\end{array}\right] .
\end{aligned}
$$

Thus, we have

$$
\begin{aligned}
\hat{x}_{t+1 / t+1}= & A_{t+1}^{1}\left\{\hat{x}_{t / t}+\left(P_{t}^{(1,1)} C_{t}^{T}-H_{t} R_{t}\right)\right. \\
\times & \left.\left(C_{t} P_{t} C_{t}^{T}+R_{t}\right)^{-1}\left(y_{t}-C_{t} \hat{x}_{t / t}\right)\right\} \\
& +H_{t+1} y_{t+1}+T_{t+1} B_{t} u_{t}
\end{aligned}
$$

and

$$
\begin{aligned}
\hat{\theta}_{t+1 / t+1}=\hat{\theta}_{t / t} & +P_{t}^{(2,1)} C_{t}^{T}\left(C_{t} P_{t} C_{t}^{T}+R_{t}\right)^{-1} \\
& \times\left(y_{t}-C_{t} \hat{x}_{t / t}\right) .
\end{aligned}
$$

Here, we used the equalities

$$
\begin{aligned}
\widetilde{C_{t}} & \widetilde{P}_{t}{\widetilde{C_{t}}}^{T}+R_{t} \\
& =\left[\begin{array}{ll}
C_{t} & O
\end{array}\right]\left[\begin{array}{ll}
P_{t}^{(1,1)} & P_{t}^{(1,2)} \\
P_{t}^{(2,1)} & P_{t}^{(2,2)}
\end{array}\right]\left[\begin{array}{c}
C_{t}^{T} \\
O
\end{array}\right]+R_{t} \\
& =C_{t} P_{t} C_{t}^{T}+R_{t}
\end{aligned}
$$

and

$$
\begin{aligned}
\widetilde{G_{t}} & =\left(\widetilde{P_{t}}\left[\begin{array}{c}
C_{t}^{T} \\
O
\end{array}\right]-\widetilde{H_{t}} R_{t}\right)\left(\widetilde{C_{t}} \widetilde{P}_{t}{\widetilde{C_{t}}}^{T}+R_{t}\right)^{-1} \\
= & \left(\left[\begin{array}{cc}
P_{t}^{(1,1)} & P_{t}^{(1,2)} \\
P_{t}^{(2,1)} & P_{t}^{(2,2)}
\end{array}\right]\left[\begin{array}{c}
C_{t}^{T} \\
O
\end{array}\right]-\left[\begin{array}{c}
H_{t} \\
O
\end{array}\right] R_{t}\right) \\
& \times\left(\widetilde{C_{t}} \widetilde{P}_{t}{\widetilde{C_{t}}}^{T}+R_{t}\right)^{-1} \\
& =\left[\begin{array}{c}
P_{t}^{(1,1)} C_{t}^{T}-H_{t} R_{t} \\
P_{t}^{(2,1)} C_{t}^{T}
\end{array}\right]\left(C_{t} P_{t} C_{t}^{T}+R_{t}\right)^{-1} \cdot(3
\end{aligned}
$$


Thus, equalities (27)-(28) can be obtained from (33) due to $\hat{\theta}_{t / t}=\hat{x}_{k / t}$.

By using the notation $\widetilde{M}_{t}$ for the augmented system (23)-(24)which corresponds to the matrix $M_{t}$ in Proposition 2.2 , we have

$$
\begin{aligned}
& \widetilde{M}_{t}=\left[\begin{array}{ll}
M_{t}^{(1,1)} & M_{t}^{(1,2)} \\
M_{t}^{(2,1)} & M_{t}^{(2,2)}
\end{array}\right] \\
& =\widetilde{P}_{t}-\widetilde{G_{t}}\left(\widetilde{C_{t}} \widetilde{P}_{t}-R_{t}\left[\begin{array}{ll}
H_{t}{ }^{T} & O
\end{array}\right]\right) \\
& =\left[\begin{array}{ll}
P_{t}^{(1,1)} & P_{t}^{(1,2)} \\
P_{t}^{(2,1)} & P_{t}^{(2,2)}
\end{array}\right]-\left[\begin{array}{c}
P_{t}^{(1,1)} C_{t}^{T}-H_{t} R_{t} \\
P_{t}^{(2,1)} C_{t}^{T}
\end{array}\right] \\
& \times\left(C_{t} P_{t} C_{t}^{T}+R_{t}\right)^{-1}\left(\left[\begin{array}{ll}
C_{t} & O
\end{array}\right]\left[\begin{array}{ll}
P_{t}^{(1,1)} & P_{t}^{(1,2)} \\
P_{t}^{(2,1)} & P_{t}^{(2,2)}
\end{array}\right]\right. \\
& \left.-\left[\begin{array}{ll}
R_{t} H_{t}^{T} & O
\end{array}\right]\right) \text {. }
\end{aligned}
$$

Thus, we have

$$
\begin{gathered}
M_{t}^{(1,1)}=P_{t}^{(1,1)}-\left(P_{t}^{(1,1)} C_{t}^{T}-H_{t} R_{t}\right) \\
\times\left(C_{t} P_{t} C_{t}^{T}+R_{t}\right)^{-1}\left(C_{t} P_{t}^{(1,1)}-R_{t} H_{t}^{T}\right), \\
M_{t}^{(1,2)}=P_{t}^{(1,2)}-\left(P_{t}^{(1,1)} C_{t}^{T}-H_{t} R_{t}\right) \\
\quad \times\left(C_{t} P_{t} C_{t}^{T}+R_{t}\right)^{-1} C_{t} P_{t}^{(1,2)}, \\
M_{t}^{(2,1)}=P_{t}^{(2,1)}-P_{t}^{(2,1)} C_{t}^{T}\left(C_{t} P_{t} C_{t}^{T}+R_{t}\right)^{-1} \\
\times\left(C_{t} P_{t}^{(1,1)}-R_{t} H_{t}^{T}\right),
\end{gathered}
$$

and

$$
\begin{aligned}
M_{t}^{(2,2)}= & P_{t}^{(2,2)}-P_{t}^{(2,1)} C_{t}^{T}\left(C_{t} P_{t} C_{t}^{T}+R_{t}\right)^{-1} \\
& \times C_{t} P_{t}^{(1,2)} .
\end{aligned}
$$

It follows from (17) in Proposition 2.2 that

$$
\begin{aligned}
& \widetilde{P_{t+1}}=\widetilde{A_{t+1}^{1}} \widetilde{M_{t}} \widetilde{A_{t+1}^{1}}{ }^{T}+\widetilde{T_{t+1}} \widetilde{J_{t+1}} Q_{t+1} \\
& \times \widetilde{J_{t+1}} \widetilde{T_{t+1}}+\widetilde{H_{t+1}} R_{t+1} \widetilde{H_{t+1}} T \\
& =\left[\begin{array}{cc}
A_{t+1}^{1} & O \\
O & I
\end{array}\right]\left[\begin{array}{ll}
M_{t}^{(1,1)} & M_{t}^{(1,2)} \\
M_{t}^{(2,1)} & M_{t}^{(2,2)}
\end{array}\right]\left[\begin{array}{cc}
A_{t+1}^{1}{ }^{T} & O \\
O & I
\end{array}\right] \\
& +\left[\begin{array}{cc}
T_{t+1} & O \\
O & I
\end{array}\right]\left[\begin{array}{l}
I \\
O
\end{array}\right] Q_{t+1}\left[\begin{array}{ll}
I & O
\end{array}\right]\left[\begin{array}{cc}
T_{t+1}^{T} & O \\
O & I
\end{array}\right] \\
& +\left[\begin{array}{c}
H_{t+1} \\
O
\end{array}\right] R_{t+1}\left[H_{t+1}^{T} O\right] .
\end{aligned}
$$

Equalities (29)-(30) follow from (38)-(40). Finally, we have equalities $P_{k}^{(2,1)}=P_{k}^{(2,2)}=P_{k}^{(1,1)}=P_{k}$ by the definition of $\widetilde{P_{k}}$.

Remark 3.2 Suppose that $E_{t} \equiv O$ holds for all $t$ (i.e., the unknown input term is zero) and that $H_{t} \equiv O$ for all $t$ (as in Proposition 2.4). In this case, it follows from Theorem 3.1 that

$$
\hat{x}_{t+1 / t+1}=A_{t}\left\{\hat{x}_{t / t}+P_{t} C_{t}^{T}\left(C_{t} P_{t} C_{t}^{T}+R_{t}\right)^{-1}\right.
$$

$$
\begin{aligned}
& \left.\times\left(y_{t}-C_{t} \hat{x}_{t / t}\right)\right\}+B_{t} u_{t} \\
\hat{\theta}_{t+1 / t+1}=\hat{\theta}_{t / t}+ & P_{t}^{(2,1)} C_{t}^{T}\left(C_{t} P_{t} C_{t}^{T}+R_{t}\right)^{-1} \\
& \times\left(y_{t}-C_{t} \hat{x}_{t / t}\right) \\
P_{t+1}^{(2,1)}=\left\{P_{t}^{(2,1)}-\right. & P_{t}^{(2,1)} C_{t}{ }^{T}\left(C_{t} P_{t} C_{t}{ }^{T}+R_{t}\right)^{-1} \\
& \left.\times C_{t} P_{t}\right\} A_{t}{ }^{T},
\end{aligned}
$$

and

$$
\begin{aligned}
P_{t+1}^{(2,2)}=P_{t}^{(2,2)}- & P_{t}^{(2,1)} C_{t}^{T}\left(C_{t} P_{t} C_{t}^{T}+R_{t}\right)^{-1} \\
& \times C_{t} P_{t}^{(2,1)^{T}} .
\end{aligned}
$$

Here, we note that the state estimate $\hat{x}_{t+1 / t+1}$ reduces to the state estimate $\hat{x}_{t+1 / t}$ in [19] when $H_{t} \equiv O$ holds. Moreover, Equalities (37)-(40) with the state estimates $\hat{x}_{t+1 / t+1}$ and $\hat{x}_{t / t}$ replaced respectively by $\hat{x}_{t+1 / t}$ and $\hat{x}_{t / t-1}$ are identical to those for the pair of the standard Kalman filter and the optimal fixed-point smoother in [19].

Let us introduce some notations:

$$
\begin{aligned}
& \nu_{t}=y_{t}-C_{t} \hat{x}_{t / t}, \\
& L_{t}=A_{t+1}^{1}\left(I-G_{t} C_{t}\right), \\
& \Psi(t, \tau)= \begin{cases}L_{t-1} L_{t-2} \cdots L_{\tau} & , t>\tau \\
I & , t=\tau,\end{cases}
\end{aligned}
$$

where the matrix $G_{t}$ was defined by (18), i.e.,

$$
G_{t}=\left(P_{t} C_{t}^{T}-H_{t} R_{t}\right)\left(C_{t} P_{t} C_{t}^{T}+R_{t}\right)^{-1} .
$$

We then have the following results due to (27).

Corollary 3.3 We have the equalities:

$$
\begin{aligned}
& \hat{x}_{k / t+1}=\hat{x}_{k / k}+\sum_{i=k}^{t} D_{i}(k) \nu_{i} \\
& =\hat{x}_{k / k}+P_{k} \sum_{i=k}^{t} \Psi(i, k)^{T} C_{i}^{T}\left(C_{i} P_{i} C_{i}^{T}+R_{i}\right)^{-1} \nu_{i} .
\end{aligned}
$$

Proof It is straightforward to show the first equality from (27). For the second equality, it is sufficient to prove the equality

$$
D_{t}(k)=P_{k} \Psi(t, k)^{T} C_{t}^{T}\left(C_{t} P_{t} C_{t}^{T}+R_{t}\right)^{-1}
$$

for $t \geq k$. By virtue of (46), equality (29) can be rewritten as

$P_{t}^{(2,1)}=P_{t-1}^{(2,1)}\left(I-C_{t-1}{ }^{T} G_{t-1}{ }^{T}\right) A_{t}^{1^{T}}=P_{t-1}^{(2,1)} L_{t-1}{ }^{T}$.

By using this equality recursively, we have

$$
\begin{aligned}
P_{t}^{(2,1)} & =P_{t-2}^{(2,1)} L_{t-2}{ }^{T} L_{t-1}{ }^{T}=\cdots \\
& =P_{k}^{(2,1)} L_{k}{ }^{T} L_{k+1}{ }^{T} \cdots L_{t-1}{ }^{T} \\
& =P_{k} \Psi(t, k)^{T} .
\end{aligned}
$$


Substituting this equality into (28), we obtain

$$
D_{t}(k)=P_{k} \Psi(t, k)^{T} C_{t}^{T}\left(C_{t} P_{t} C_{t}^{T}+R_{t}\right)^{-1},
$$

i.e., $(50)$.

Finally, we study the reduction of the estimation error by the fixed-point smoothing over the optimal filtering. Due to (27), we have

$$
P_{t}^{(2,2)}=\mathbb{E}\left[\left(x_{k}-\hat{x}_{k / t}\right)\left(x_{k}-\hat{x}_{k / t}\right)^{T}\right] .
$$

Denote this matrix simply by $P_{k / t}$. It then follows from (30) that

$$
P_{k / t+1}=P_{k / t}-P_{t}^{(2,1)} C_{t}^{T}\left(C_{t} P_{t} C_{t}^{T}+R_{t}\right)^{-1} C_{t} P_{t}^{(2,1)^{T}}
$$

Summing up these equalities for $t=k, k+1, \cdots, s$, we have

$$
\begin{aligned}
& P_{k / k}-P_{k / s+1} \\
& =\sum_{i=k}^{s} P_{i}^{(2,1)} C_{i}^{T}\left(C_{i} P_{i} C_{i}^{T}+R_{i}\right)^{-1} C_{i} P_{i}^{(2,1)^{T}} .
\end{aligned}
$$

Thus, the right hand side indicates the amount of the reduction of the estimation error by the fixed-point smoothing over the optimal filtering.

\section{Conclusion}

In this paper, the fixed-point smoothing problem for a discrete-time linear stochastic system with unknown disturbances has been considered. A new smoothing algorithm which is robust to unknown disturbances has been derived from the optimal filter for linear systems with unknown inputs in the author's previous work. It has been shown that this algorithm reduces to the well known optimal smoother derived from the Kalman filter when unknown inputs disappear.

\section{Acknowledgements}

The author wishes to thank Professor Yuichi Sawada of Kyoto Institute of Technology for his help during preparation of this paper, and to thank anonymous referees for their insightful comments which improved the earlier version of this paper.

\section{References}

[1] B. D. O. Anderson and J. B. Moore, Optimal Filtering, Prentice-Hall, Englewood Cliffs, NJ, 1979.

[2] A. E. Bryson, Jr. and Y. C. Ho, Applied Optimal Control, Blaisdell Publishing Company, Waltham, Massachusetts, 1969.
[3] F. Caliskan, H. Mukai, N. Katz and A. Tanikawa, "Game estimators for air combat games with unknown enemy inputs", Proc. American Control Conference, Denver, Colorado, pp. 5381-5387 (2003)

[4] S. Chang and P. Hsu, "State estimation using general structured observers for linear systems with unknown input", Proc. 2nd European Control Conference: ECC'93, Groningen, Holland, pp. 1794-1799 (1993).

[5] J. Chen and R. J. Patton, "Optimal filtering and robust fault diagnosis of stochastic systems with unknown disturbances", IEE Proc. of Control Theory Applications Vol. 143, No. 1, pp. 3136 (1996).

[6] J. Chen and R. J. Patton, Robust Model-based Fault Diagnosis for Dynamic Systems, Kluwer Academic Publishers, Norwell, Massachusetts, 1999.

[7] J. Chen, R. J. Patton and H. -Y. Zhang, "Design of unknown input observers and robust fault detection filters", Int. J. Control Vol. 63, No. 1, pp. 85-105 (1996).

[8] M. Darouach, M. Zasadzinski and J. Y. Keller, "State estimation for discrete systems with unknown inputs using state estimation of singular systems", Proc. American Control Conference, pp. 3014-3015 (1992).

[9] M. Darouach, M. Zasadzinski, O. A. Bassang and S. Nowakowski, "Kalman filtering with unknown inputs via optimal state estimation of singular systems", Int. J. Systems Science Vol. 26, pp. 20152028 (1995).

[10] P. M. Frank, "Fault diagnosis in dynamic system using analytical and knowledge based redundancy: a survey and some new results", Automatica Vol. 26, No. 3, pp. 459-474 (1990).

[11] M. Hou and P. C. Müller, "Unknown input decoupled Kalman filter for time-varying systems", Proc. 2nd European Control Conference: ECC'93, Groningen, Holland, pp. 2266-2270 (1993).

[12] M. Hou and P. C. Müller, "Disturbance decoupled observer design: a unified viewpoint", IEEE Trans. Automatic Control Vol. 39, No. 6, pp. 1338-1341 (1994).

[13] M. Hou and R. J. Patton, "Optimal filtering for systems with unknown inputs", IEEE Trans. Automatic Control Vol. 43, No. 3, pp. 445-449 (1998).

[14] T. Kailath, "A view of three decades of linear filtering theory", IEEE Trans. Inform. Theory Vol. 20, No. 2, pp. 146-181 (1974). 
[15] T. Kailath, "Supplement to a survey to data smoothing", Automatica Vol. 11, No. 11, pp. 109111 (1975).

[16] T. Kailath, Lectures on Linear Least-Squares Estimation, Springer, 1976.

[17] R. E. Kalman, "A new approach to linear filtering and prediction problems", in Trans. ASME, J. Basic Eng. Vol. 82D, No. 1, pp. 34-45 (1960).

[18] R. E. Kalman, "New methods in Wiener filtering theory", in Proc. of First Symp. Eng. Appl. of Random Function Theory and Probability (J. L. Bogdanoff and F. Kozin, eds.), Wiley, pp. 270-388, (1963).

[19] T. Katayama, Applied Kalman Filtering, New Edition, in Japanese, Asakura-Shoten, Tokyo, Japan, 2000 .

[20] J. S. Meditch, "A survey of data smoothing for linear and nonlinear dynamic systems", Automatica Vol. 9, No. 2, pp. 151-162 (1973).

[21] R. J. Patton, P. M. Frank and R. N. Clark, Fault Diagnosis in Dynamic Systems: Theory and Application, Prentice Hall, 1996.

[22] Y. Sawada and A. Tanikawa, "Optimal filtering and robust fault diagnosis of stochastic systems with unknown inputs and colored observation noises", in Proc. 5th IASTED Conf. Decision and Control, Tsukuba, Japan, pp. 149-154, (2002).

[23] A. Tanikawa and Y. Sawada, "Minimum variance state estimators with disturbance decoupling property for optimal filtering problems with unknown inputs", in Proc. of the 35th ISCIE Int. Symp. on Stochastic Systems Theory and Its Appl., Ube, Japan, pp. 96-99, (2003). 